\title{
ANÁLISIS DE LA LEY ORGÁNICA DE DISCAPACIDADES Y OTRAS DISPOSICIONES COMO APOYO A LOS PROCESOS DE INCLUSIÓN
}

\section{ANALYSIS OF THE CURRENT DISABILITY LAW AND OTHER PROVISIONS IN SUPPORT OF INCLUSION PROCESSES}

\author{
GINA MARCELA VALDIVIESO MIÑO ${ }^{1}$ \\ RUTH MAGDALENA LALAMA ROVAYO ${ }^{2}$
}

Recibido: 15 de mayo de 2017 Aceptado: 29 de agosto de 2017

\footnotetext{
${ }^{1}$ Pontificia Universidad Católica del Ecuador, Facultad de Psicología, Quito, Ecuador (gvaldivieso@puce.edu.ec).

${ }^{2}$ Universidad de las Américas, Facultad de Educación, Quito, Ecuador (ruth.lalama@udla.edu.ec)
} 



\title{
ANÁLISIS DE LA LEY ORGÁNICA \\ DE DISCAPACIDADES Y OTRAS DISPOSICIONES COMO APOYO A LOS PROCESOS DE INCLUSIÓN
}

\section{ANALYSIS OF THE CURRENT DISABILITY LAW AND OTHER PROVISIONS IN SUPPORT OF INCLUSION PROCESSES}

\author{
Gina Marcela Valdivieso Miño y Ruth Magdalena Lalama Rovayo
}

Palabras clave: Discapacidad, inclusión, barreras, ámbito jurídico en discapacidades Key Word: Disability, inclusion, barriers, laws for disability issues

\section{RESUMEN}

Las personas con discapacidad (PCD) han estado desprotegidas por muchos años, y en muchos casos excluidas y discriminadas, lo que ha generado una baja autoestima en las mismas. Con el estudio realizado, se procura dar a conocer la existencia y disposiciones contenidas en la legislación que pretenden, establecer acciones directas que favorezcan la inclusión y bienestar de las PCD. Si bien el Ecuador ha avanzado en este tema a nivel político y jurídico, aún se observa contradicciones entre ellas. El momento que la PCD quiere ejercer sus derechos, la falta de información y el sinnúmero de procedimientos imposibilitan su acceso a los mismos. Pretenderemos describir el marco conceptual de qué se entiende por discapacidad y además, realizar un análisis bibliográfico del marco jurídico que rige en el país, para finalmente contrastarlo con 
la realidad ecuatoriana, los servicios dispuestos, responderse a la pregunta planteada acerca de la complejidad que se presenta para una persona con discapacidad que desea acceder a los servicios requeridos y emitir las conclusiones y recomendaciones para futuras investigaciones sobre este tema.

\section{ABSTRACT}

People with disabilities, PWD, have been postponed for many years, and in some cases excluded and discriminated, which leads to low self-esteem. With this study, the aim is to share information about the existence and legal dispositions that pretend to establish direct actions that favor inclusion and wellbeing of people with disabilities. It is true that in Ecuador this topic has advanced al political and legal aspects, there are still contradictions. The moment that PWD want to exercise their rights, there is a lack of information and the amount of procedures makes it difficult to access theses rights. We will pretend to describe the conceptual framework of what is a disability and on the other hand analyze the legal foundation, and finally contrast the Ecuadorian reality and elaborate conclusions and recommendations for future investigations about this topic.

\section{INTRODUCCIÓN}

"La discapacidad forma parte de la condición humana".

(Verdugo M.A. Schalock R. , 2013)

Es evidente que una sociedad es diversa y compleja, esto exige comprender las dimensiones de lo que representa para un Estado asumir el reto de la protección y atención a las personas con discapacidad - PCD. La inclusión de estas personas ha recorrido un largo camino; iniciando con su exclusión total e inclusive en muchos casos su extermi- nio, pasando por la segregación y separación de los grupos o de la participación familiar, hasta llegar a la integración en varios campos, sin embargo, la inclusión, está aún en proceso.

Al referirnos a inclusión, se toma en cuenta la valoración de las diferencias como una posibilidad de crecimiento, justicia y solidaridad, valores que hacen 
más grande a una nación. Implica además el respeto a los derechos en todos los ámbitos del desarrollo humano. No es sinónimo de igualdad, sino de equidad, lo que significa que se debe atender a las necesidades individuales de las personas, sin olvidar la garantía de su participación libre en la comunidad. De esta manera, toda sociedad democrática debe favorecer el crecimiento y el desarrollo de todos sus habitantes con la aprobación de leyes y acuerdos que garanticen sus derechos. (Verdugo M.A. Schalock R. , 2013).

Las barreras existentes impiden que las personas con discapacidad puedan desarrollar un plan de vida y alcanzar logros. Estas barreras son de todo tipo; entre ellas arquitectónicas, actitudinales, educativas y culturales. La eliminación de estas barreras tendrá como resultado el ejercicio de derechos inherentes a todo ser humano en el ámbito laboral, educativo, social, emocional, de recreación y vivienda. (Convención de derechos de las personas con discapacidad, 2006).

En los últimos años, la discapacidad ha sido objeto de atención y análisis y por tanto se ha invertido en proyectos y planes que mejoren las condiciones de vida de PCD. (Verdugo A. M., 1998).

En el Ecuador, también se han reforzado leyes que permitan el acceso a la sociedad a las PCD, sin embargo, es fácil suponer que estas leyes aún sean desconocidas por un amplio grupo de la comunidad. Por ello, el tener referencias acerca de los procesos de la aplicación de dichas leyes y acuerdos para el apoyo a la inclusión, permite constatar si en efecto las PCD tienen acceso a la participación total, y cuáles son los organismos centrales a los que deben acudir y amparar sus derechos. Por ello, el presente artículo bibliográfico pretende ser una herramienta en los campos relacionados.

En el Ecuador la preparación de las leyes en torno a la discapacidad inicia en 1940, en donde se establece en la Constitución de la República del Ecuador - CRE, la cual, en ese entonces, en el art. 27 mencionaba: "el acceso a la educación de todos los ciudadanos sin discriminación alguna". (Asamblea Nacional del Ecuador, 2008).

En la Ley Orgánica de Educación en 1945, se disponía que la atención se deba dar a todos los niños y niñas que se conociera adolecen anormalidades tanto biológicas como mentales. Es necesario entonces pensar hasta qué punto esta ley se ha cumplido en cuanto a la inclusión de los niños con discapacidad en las escuelas e instituciones públicas o privadas del Ecuador.

Las necesidades de las PCD han sido por mucho tiempo olvidadas, convirtiendo a este grupo humano en una especie de seres "invisibles" a quienes no se les ha tomado en cuenta en las 
decisiones públicas ni políticas. "El desconocimiento y el menosprecio de los derechos humanos han originado actos de barbarie ultrajantes para la conciencia de la humanidad". (Asamblea Nacional, Registro Oficial N. 796, 2012).

El Ecuador ha hecho avances importantes en el campo legislativo, es así que, en el año de 1992, se creó el Consejo Nacional para las Discapacidades -CONADIS (con el ánimo de llevar a cabo los procesos de inclusión social, económica y cultural de las personas con discapacidad, organismo que en el 2012 cambia al nombre de Consejo Nacional para la Igualdad de Discapacidades). Este se basa en la Convención sobre los Derechos de las Personas con Discapacidad de Naciones Unidas, en Ley 42 de 27 de agosto de 1999 sobre Equiparación de Oportunidades para las personas con discapacidad y en la Ley 23 de 28 de junio de 2007. (Asamblea Nacional, Registro Oficial N. 796, 2012).

La puesta en marcha de leyes y proyectos destinados a la inclusión social y educativa en el Ecuador, ha cobrado fuerza en la última década. Existe una correlación entre diversos ministe- rios y organismos que la favorecen y se establecen los beneficios que el Estado brinda a las PCD.

En este artículo se realiza un compendio de algunas de las leyes, políticas, procedimientos y buenas prácticas que se han emitido en el pasar de los años, estableciendo cronológicamente los avances que se han alcanzado, fundamentalmente en las últimas décadas.

Esta información, da la posibilidad de conocer los lugares y documentos a donde referirse en términos de derechos y deberes de las PCD. Quienes tienen familiares, trabajan con PCD o presentan alguna discapacidad, deben tener fácil acceso a la información para lograr una mayor inclusión y por tanto participación en todas las actividades relacionadas con sus intereses en la vida personal y en la sociedad para ejercer los derechos e instar a que las organizaciones cumplan con sus obligaciones. A lo largo del presente artículo se puede evidenciar que hay información poco consistente en las distintas fuentes consultadas. Se tomarán en cuenta las proporcionadas por las páginas oficiales. 


\section{MARCO CONCEPTUAL}

\section{Enfoque histórico en torno a la discapacidad}

Para una noción de discapacidad es necesario exponer distintos conceptos y perspectivas a lo largo de la historia.

De acuerdo con lo que indica Toboso (2008), hasta el siglo XVII, se entendía a la discapacidad como un castigo relacionado con las creencias religiosas, generando rechazo y considerándose una carga para la sociedad. A este modelo de concebir la discapacidad se lo denomina de "prescindencia". Esto condujo a una etapa eugenésica que eliminaba a los niños y niñas con discapacidad y luego en la edad media a la marginación (Toboso, 2008).

Más adelante, se desarrolla un modelo de rehabilitación, que "centraba el problema en la persona, en sus deficiencias, limitaciones y dificultades" (Verdugo M.A. Schalock R., 2013, p.17). Se inicia así un proceso de rehabilitación que representa un avance en la atención a la discapacidad, sin embargo la PCD no es incluida en la sociedad. Se forman instituciones y organismos destinados a tratar las dificultades, Verdugo sostiene que aún persiste este modelo incluso con ciertas actitudes del modelo tradicional. (Verdugo M.A. Schalock R., 2013).
El modelo que nace como rechazo a las dos anteriores es un modelo social o como lo llama Verdugo (2013), un modelo o paradigma de la autonomía personal. Se ve a la discapacidad como un problema del entorno y no de la persona. Favorece la autonomía en la búsqueda de la autodeterminación personal. Se considera que las personas con discapacidad pueden llegar a ser un aporte para la sociedad y por lo tanto esta concepción genera un impacto, pues se comprende que las personas están en capacidad de llevar el control de sus vidas (Toboso, 2008). Esta perspectiva de la discapacidad genera una sociedad distinta, democrática y consciente del valor que reside en las diferencias.

\section{Definición de discapacidad}

De acuerdo con la OMS (Organización Mundial de la Salud, 2016), "Discapacidad es un término general que abarca las deficiencias, las limitaciones de la actividad y las restricciones de la participación." Tomando en cuenta esta definición y relacionándola con el modelo de autonomía personal, la discapacidad en efecto se hace más evidente en cuanto mayores sean las barreras y restricciones que tengan las PCD para poder realizar las actividades que el resto de la socie- 
dad ejerce.

¿Quién es una persona con discapacidad?

En la Convención Internacional sobre los derechos de las personas con discapacidad. Artículo 1. Propósito. P4: (Convención de derechos de las personas con discapacidad, 2006), se señala que "Personas con discapacidad son aquellas que tienen deficiencias físicas, mentales, intelectuales o sensoriales a largo plazo que, al interactuar con diversas barreras, pueden impedir su participación plena y efectiva en la sociedad, en igualdad de condiciones con los demás." Además de definir conceptos importantes, en esta convención se llegaron a acuerdos que garanticen la inversión en programas de apoyo y desarrollo, así como asegurar la participación de las PCD.

\section{Terminología}

Debido a que la concepción de la discapacidad depende de varios factores, entre ellos, sociales, culturales e individuales, persiste en nuestro medio el manejo de términos poco apropiados para referirse a la discapacidad. Las formas de referirse a la discapacidad han sido variadas y por mucho tiempo discriminatorias y peyorativas.

En 1980, la Organización Mundial de la Salud elaboró un documento de clasificación conocido como "Clasificación Internacional de Deficiencias, Discapacidades y Minusvalías" - (CIDDM). Más adelante, en el año 2001 se hizo una revisión a este documento y debido a los nuevos enfoques se elabora el CIF, Clasificación Internacional del Funcionamiento, de la Discapacidad y de la Salud (OMS - OPS, 2001). A continuación, la tabla 1, que describe los términos y ejemplos:

\section{Tabla 1. Terminología}

\begin{tabular}{|c|c|c|}
\hline Término & Descripción & Ejemplo \\
\hline Deficiencias & $\begin{array}{l}\text { Pérdida o anomalía en alguna estructura } \\
\text { corporal o psicológica. }\end{array}$ & Pérdida auditiva \\
\hline Minusvalía & $\begin{array}{l}\text { Limitación en el desempeño de un rol } \\
\text { como consecuencia de una deficiencia } \\
\text { que tiene }\end{array}$ & $\begin{array}{l}\text { Este término ya no se emplea por la } \\
\text { connotación peyorativa. }\end{array}$ \\
\hline $\begin{array}{l}\text { Limitaciones en } \\
\text { la Actividad }\end{array}$ & $\begin{array}{l}\text { Dificultades que se tienen para la } \\
\text { realización de alguna actividad. }\end{array}$ & $\begin{array}{l}\text { Una persona en silla de ruedas no } \\
\text { puede acceder a un edificio por falta } \\
\text { de rampas. }\end{array}$ \\
\hline Actividad & Es la ejecución de una tarea o acción & Estudiar, votar, comprar \\
\hline Barreras & $\begin{array}{l}\text { Limitaciones al acceso a beneficios y } \\
\text { derechos de todo ser humano }\end{array}$ & $\begin{array}{l}\text { Arquitectónicas, culturales, sociales, } \\
\text { actitudinales, de lenguaje y } \\
\text { comunicación, curriculares, entre } \\
\text { otras. }\end{array}$ \\
\hline
\end{tabular}

Elaborado por: Las autoras 


\section{Integración versus Inclusión}

Para analizar la diferencia de estos términos se ha definido a la integración e inclusión de la siguiente manera: la integración se centra en la discapacidad o enfermedad, por lo que se vuelve un sistema paternal. Mientras que en la inclusión se enfoca en la persona como poseedora de derechos. Es la sociedad la que se adapta, no la persona. En ese sentido, la inclusión favorece el desa- rrollo de las PCD en sus propias capacidades y proyectos de vida. Se entiende en procesos inclusivos que las personas tienen derechos de acceder a todos los servicios de salud y educación. Se contempla la participación total en todos los aspectos de la sociedad sin discriminación. La ilustración que se presenta de inmediato, es una representación gráfica parafraseada:

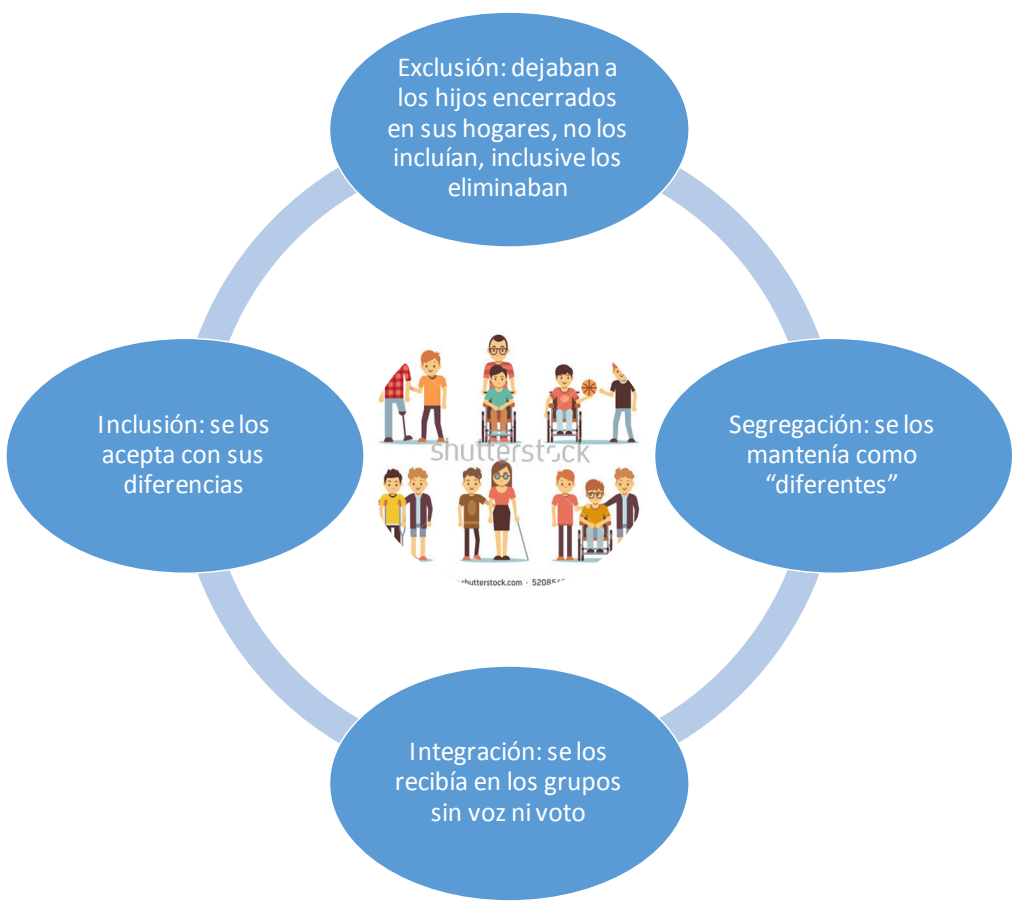

Gráfico 1. Explicación de avances en el término

Elaborado por las autoras, basadas en el gráfico tomado de internet: www.shutterstock.com 


\section{Clasificación de la discapacidad}

Es necesario definir los tipos de discapacidad con los que se puede encontrar, dentro de la clasificación que da el gobierno, que de acuerdo al compendio trabajado por la (CEPAL - Bernal M.E. y Valencia C., 2016) se basa en: "El Sistema Único de Calificación de Discapacidad en el Ecuador reconoce las siguientes discapacidades (CONADIS y SENPLADES, 2013, p 22), en donde se clasifica a las personas con discapacidad en física, cognitiva, sensorial e intelectual.

En el año 2012, la tipología reconocida por el Sistema Único de Calificación de Discapacidad en el Ecuador corresponde al detalle del siguiente gráfico:

Gráfico 2. Clasificación y descripción de las discapacidades

\begin{tabular}{|l|l|}
\hline FISICA & PSICOLOGICA \\
\hline INTELECTUAL & DE LENGUAJE \\
\hline VISUAL & AUDITIVA \\
\hline
\end{tabular}

Elaborado por: Las autoras

Tomado de: (Agenda-Nacional-para-Discapacidades, 2016) 


\section{MARCO JURÍDICO}

Para una mayor facilidad de com- siguiente gráfico que indica el orden de prensión de las leyes que fueron revisa- prelación utilizado, conforme la pirámide das en este documento, se presenta el de Kelsen:

Gráfico 3: Pirámide organizativa de documentos jurídicos en Ecuador

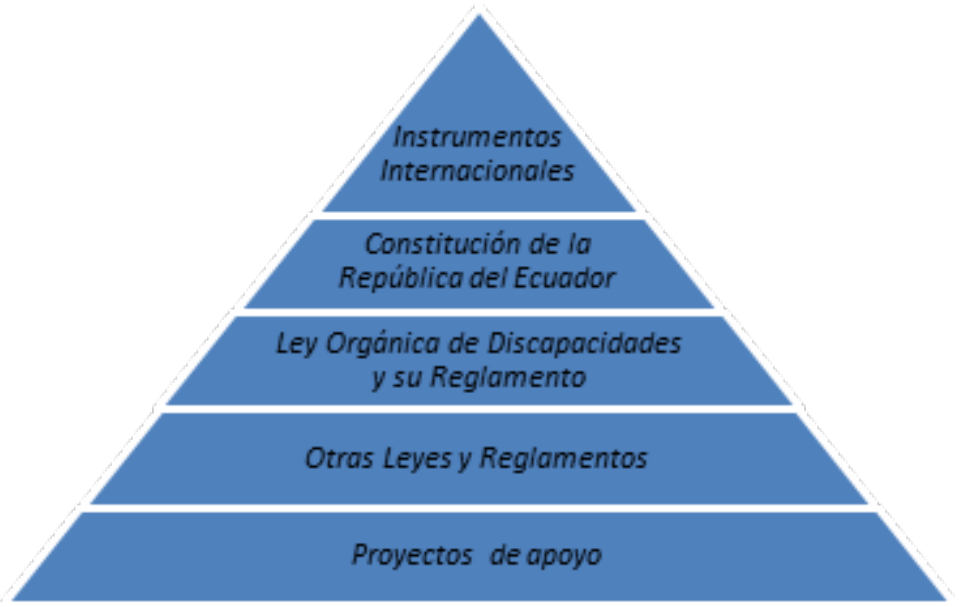

Elaborado por: Las autoras

\section{Declaración Universal de los Derechos Humanos}

En el año de 1948, la Asamblea General de la Naciones Unidas (ONU) en la resolución 217-A, redactó lo que sería la base de los derechos de todas las personas. Son treinta artículos que garantizan el respeto a la vida, a las libertades y a la dignidad humana. Este acuerdo fue firmado por los Estados Miembro de la ONU, entre ellos Ecuador. En sus afir- maciones indica: "La libertad, la justicia y la paz en el mundo tienen por base el reconocimiento de la dignidad inherente a todos los miembros de la familia humana y de sus derechos iguales e inalienables". (Organización de las Naciones Unidas, s.d.).

En el año 2006, se llevó a cabo la Convención Internacional sobre los Derechos de las PCD. Esta convención marcó un avance importante en la revisión 
de las igualdades al definir leyes que se vinculan y considerar como sujetos de derecho a las personas con discapacidad (Verdugo M.A. Schalock R., 2013).

Como resultado de esta convención se elaboraron 50 artículos, de los cuáles 26 se refieren a "obligaciones específicas de los estados" (Verdugo M.A. Schalock R., 2013, p. 34), esto quiere decir que se priorizan derechos agrupados en aspectos concretos conforme a libertades fundamentales, tales como la vida independiente, la autonomía, derecho a educación, salud, recreación, vivienda y trabajo, entre otros.
En el Ecuador, la aplicación de los derechos humanos a lo largo de la historia no se ha llevado a cabo de manera justa o equitativa. Con el respaldo de la Declaración de los Derechos Humanos, la última década ha buscado reducir barreras de la sociedad, introduciendo cambios importantes en algunos de los estamentos jurídicos que la representan.

A nivel mundial, se han establecido acuerdos y compromisos para que los países asuman responsabilidades a favor de una educación para todos. En la tabla 2 se enumeran los más importantes:

\section{Tabla 2. Acuerdos mundiales}

\begin{tabular}{|l|l|}
\hline Año & Tema \\
\hline 1948 & Declaración Universal de los Derechos Humanos, (art. 26) \\
\hline 1978 & Informe Warnock, elaborado por Mary Warnock \\
\hline 1982 & Programa de Acción Mundial para los Impedidos, aprobado por la Asamblea General de las Naciones Unidas \\
\hline 1990 & $\begin{array}{l}\text { "Satisfacción de las Necesidades Básicas del Aprendizaje" fue parte de la Declaración Mundial sobre Educación Para } \\
\text { Todos (EPT) efectuada en Jomtien, Tailandia }\end{array}$ \\
\hline 1992 & $\begin{array}{l}\text { Se define el } 3 \text { de diciembre de cada año como el "Día Internacional de los Impedidos", en la Asamblea General de las } \\
\text { Naciones Unidas }\end{array}$ \\
\hline 2000 & $\begin{array}{l}\text { Surge el concepto de "Escuela Integradora": "Acceso y Calidad", como Declaración de Salamanca en la conferencia } \\
\text { Mundial sobre Necesidades Educativas Especiales - NEE }\end{array}$ \\
\hline 2006 & Dakar 2000, se reafirma la idea de la Declaración Mundial sobre Educación para Todos (Jomtien, 1990), en el Foro \\
\hline
\end{tabular}




\section{Constitución de la República del Ecuador}

El Ecuador, en esta línea ha desarrollado articulados que garantizan los derechos y ejecución a favor de las PCD. Es así que, dentro de la Constitución de la República del Ecuador se incluyen en varios de sus artículos la obligación de todos los ecuatorianos a ejercer derechos y deberes, independiente de su condición.

El artículo 11 numeral 2 señala que:

"Todas las personas son iguales y gozarán de los mismos derechos, deberes y oportunidades... La ley sancionará toda forma de discriminación". (Asamblea Nacional del Ecuador, 2008).

Así mismo, en su artículo 16 numeral 4, queda establecido el acceso y uso de todas las formas de comunicación visual, auditiva, sensorial y otras que permitan la inclusión de personas con discapacidad - PCD.

En la carta magna además señala que el Estado prestará especial protección a las personas en condición de doble vulnerabilidad, así como la disposición que se otorgue asistencia humanitaria, preferente y especializada. Queda de manifiesto la plena integración social de quienes tengan discapacidad y la garantía que serán incorporados en el sistema de educación regular y en la sociedad.

En los artículos 47, 48, 49, 61, 62 y 66 , se abordan temas relacionados con las PCD, promoviendo la igualdad de oportunidades, la participación intergeneracional, la opción de votar y en general el derecho a la integridad, en donde aclara que deberá darse una vida libre de violencia y la necesidad de desarrollar leyes que establezcan procedimientos especiales y expeditos para el juzgamiento y sanción.

En este documento jurídico se definen además los Consejos Nacionales de Igualdad, a los que les da la potestad de ejercer atribuciones en la formulación, transversalización, observancia, seguimiento y evaluación de las políticas públicas relacionadas con discapacidades y movilidad humana. Insta a coordinar con las entidades rectoras y ejecutoras y con los organismos especializados en la protección de los derechos de todos los niveles de gobierno. Garantiza además la inserción y accesibilidad en igualdad de condiciones al trabajo remunerado de las personas con discapacidad, prohibe disminuir la remuneración del trabajador con discapacidad por cualquier circunstancia relativa a su condición y afirma que promoverá un régimen laboral que funcione en armonía con las necesidades de cuidado humano, mencionando puntualmente entre otros a las 
PCD y que generará las condiciones para la protección integral de sus habitantes a lo largo de sus vidas, asegurando los derechos y principios reconocidos en la Constitución, así como la universalidad obligatoria para cubrir contingencias de enfermedad, discapacidad, muerte.

Asevera que las prestaciones de salud de las contingencias de enfermedad y maternidad se brindarán a través de la red pública integral de salud, se incluirá en el seguro social campesino, se ofrecerán prestaciones de salud y protección contra las contingencias de invalidez, discapacidad, vejez y muerte. En el art. 381, se afirma que el Estado protegerá, promoverá y coordinará la cultura física, que comprende el deporte y fomentará la participación de las PCD.

Finalmente, en la Disposición Transitoria Sexta indica que los Consejos Nacionales de Niñez, Adolescencia, Discapacidades, Mujeres, Pueblos y otros, se constituirán en Consejos Nacionales para la Igualdad, para lo que adecuarán su estructura y funciones a la Constitución y se definirá la ley orgánica que los ampare y su reglamento, el mismo que se aprueba y publica su confirmación en el Decreto Ejecutivo.

\section{Ley Orgánica de Discapacidades}

Como siguiente nivel en la pirámide mencionada, se encuentra la Ley Orgánica de Discapacidades - LOD y su Reglamento, tiene como fecha el 25 de septiembre del 2012 para la publicación en el Registro Oficial No. 796 de la mencionada ley y que actualmente se encuentra en vigencia. (Agenda-Nacional-para-Discapacidades, 2016. p. 30).

El 10 de agosto de 1992, a través de la ley 180, se crea el Consejo Nacional de Discapacidades - CONADIS con el ánimo de ser un ente autónomo de carácter público, con la atribución de dictar políticas, coordinar acciones y ejecutar e impulsar investigaciones referentes a las discapacidades en el país. Esta entidad fue aprobada y publicada en el Registro Oficial No 996. En mayo del 2012 por mandato constitucional se reemplaza el nombre de Consejo Nacional de Discapacidades por Consejo Nacional para la Igualdad de Discapacidades. Este organismo, se regirá por los siguientes principios rectores: igualdad, alternabilidad, participación democrática, inclusión, interculturalidad y pluralismo. (CONADIS- Normas jurídicas en Discapacidad Ecuador, 2012).

En el artículo 1 se asegura que todas las personas con discapacidad recibirán atención oportuna, así como la garantía de sus derechos. En cuanto al ámbito que abarca, se especifica en el artículo 2 que la ley ampara a todas las personas con discapacidad sin excepción alguna. El artículo 3 establece los fines de esta ley. En los siguientes 
artículos de esta ley, los temas más relevantes para este estudio tratan sobre los principios de no discriminación y las acciones afirmativas que se deben llevar a cabo, el "in dubio pro hominem", la igualdad de oportunidades, la responsabilidad social colectiva, la celeridad y eficacia, la interculturalidad, la participación e inclusión, la accesibilidad, la protección de niñas, niños y adolescentes, la atención prioritaria y otros principios consagrados en la Constitución de la República del Ecuador, la Convención de los Derechos de las PCD y demás tratados e instrumentos internacionales de derechos humanos. Cabe insistir en que esta ley ampara a toda la población de PCD y personas con deficiencia o condición discapacitante, se encuentren o no en el país.

En la sección II de la ley en mención, se detallan las entidades encargadas de las diferentes actividades a cumplir, los organismos y representaciones diplomáticas en el exterior, el proceso de calificación y la acreditación de la cédula de ciudadanía como único documento habilitante. Otros artículos de la ley hablan del registro nacional de PCD, la interconexión de las bases de datos y la remisión de datos de los recién nacidos que presenten discapacidad, guardando estricta reserva de su identidad.
En lo que corresponde a los derechos de las $P C D$, se garantiza lo dicho en la Constitución de la República del Ecuador e instrumentos internacionales e inclusive reconoce los derechos en familiares de hasta cuarto grado de consanguinidad y segundo de afinidad, que demuestren tener bajo su responsabilidad y/o cuidado a una PCD para recibir los beneficios correspondientes.

Los artículos 17 y 18 explican las medidas de acción afirmativa y cooperación internacional en cuanto a la coordinación con autoridades nacionales la asesoría técnica y los recursos destinados a la atención.

Actualmente la calificación del porcentaje y grado de discapacidad lo realiza el Ministerio de Salud, entidad que debería trabajar conjunta y coordinadamente con el CONADIS en la atención de PCD en temas de medición. Esta calificación es importante debido a que permite el acceso a beneficios que serán mencionados más adelante.

\section{Otras leyes concordantes}

A continuación, se presenta una tabla con algunas de las leyes que rigen y habilitan la participación y el acceso a los derechos que corresponden a las PCD y que se encuentran vigentes a la fecha. 


\section{Tabla 3. Acuerdos mundiales}

\begin{tabular}{|c|c|c|}
\hline LEY & Año - RO & Artículos concordantes \\
\hline CRE - Constitución de la República del Ecuador & $2008-$ & $\begin{array}{l}\text { Artículos 11, 16, 35, 36, 42, 46, 47, 48, } \\
\text { 49, 61, 62, 66, 81, 156, 330, 333, 341, } \\
369,373,381\end{array}$ \\
\hline PNBV__Plan Nacional del Buen Vivir & 2008 & $\begin{array}{l}\text { Presentación, Políticas de transferencia, } \\
\text { art } 341\end{array}$ \\
\hline $\begin{array}{l}\text { AND - Agenda Nacional para las Discapaci- } \\
\text { dades }\end{array}$ & 2014 & Documento completo \\
\hline LOD - Ley Orgánica de Discapacidades & $2012-R 0796$ & $\begin{array}{l}\text { Art. } 23,54,55,57,73,74,75,76,79,80 \text {, } \\
85\end{array}$ \\
\hline $\begin{array}{l}\text { RLOD - Reglamento de la Ley Orgánica de } \\
\text { Discapacidades }\end{array}$ & 2013 - DE 171 RO 2.S 145 & Documento Completo \\
\hline Código Penal & $\begin{array}{l}2012 \text { última modificación - R0 } \\
147 \text { de ene } 1971\end{array}$ & Art. $30,30 \mathrm{~A}$ \\
\hline Código de Procedimiento penal & $\begin{array}{l}2010 \text { última modificación R0 } 160 \\
\text { - Ley R0 } 360 \text { de ene } 2000\end{array}$ & Art. 171 \\
\hline Código del Trabajo & $\begin{array}{l}2011 \text { - última modificación a } \\
\text { Codificación R0 } 167 \text { del } 2005\end{array}$ & $\begin{array}{l}\text { Ley Reformatoria RO } 198 \text { de enero } 2006 \\
\text { / Fe de erratas de RO } 223 \text { marzo } 2006 \text { / } \\
\text { Art. 42, 79, } 152\end{array}$ \\
\hline LOSEP - Ley Orgánica de Servicio Público & 2010 - R0 294 de octubre 2010 & Art. $27,33,60,63,64$ \\
\hline $\begin{array}{l}\text { INEN - Reglamento Técnico RTE INEN } 042 \text { - } \\
\text { Accesibilidad de las PCD y movilidad reducida } \\
\text { al medio físico }\end{array}$ & $\begin{array}{l}2009 \text { - Resolución INEN 91, R0 } \\
69 \text { del } 18 \text { noviembre } 2009\end{array}$ & Art. $4-4.1$ \\
\hline Ley del Deporte & 2010 - L0 - R0 255 agosto 2010 & $\begin{array}{l}\text { Art. } 14,42,43,44,46,66,67,68,69,70 \text {, } \\
79,80,84,135,141,142\end{array}$ \\
\hline MSP - Ministerio de Salud Pública - Acuerdo & 1999 / AM- 1801 - MSP & $1,2,4,6,7,8,11,12,13$ \\
\hline Código de la Niñez y Adolescencia & $\begin{array}{l}2009 \text { última modificación al RO } \\
737 \text { de ene } 2003\end{array}$ & $\operatorname{Art} 55,78,87,103,251$ \\
\hline Ley Oegánica de Educación Intercultural & 2011 e R0 417 de marzo 2011 & $\begin{array}{l}\text { Art. 6, 47, 132, Disposiciones Generales } \\
\text { Vigésima Quinta, Trigésima Primera }\end{array}$ \\
\hline $\begin{array}{l}\text { Ley Orgánica de Transporte Terrestre Tránsito y } \\
\text { Seguridad Vial, Consejo Nacional de Aviación } \\
\text { Civil }\end{array}$ & $\begin{array}{l}2012 \text { última modificación al DE } \\
1196 \text { / } 2011 \text { - RO } 398 \text { de mayo } \\
2008 \text { / }\end{array}$ & $\begin{array}{l}\text { Art. 48, 82, } 200 \\
\text { Codificación de la Ley de Aviación Civil, } \\
\text { Art. } 55 \text { - - Distrito Metropolitano de Quito } \\
\text { - Resolución }\end{array}$ \\
\hline
\end{tabular}

Elaborado por: las autoras 
Adicionalmente existen otras leyes que mencionan a las PCD: de educación superior, de juventud, del anciano, LOCNI - Ley Orgánica de los Consejos Nacionales para la Igualdad y su reglamento, MIES - Metodología y aplicación para Análisis Costo - Beneficio de los Servicios de Cuidado para la Población con Discapacidad y Población Adulta
Mayor el Código Tributario, que pueden ser revisadas en un próximo análisis.

En cuanto a la atención en los distintos ámbitos de desarrollo en la sociedad; salud, educación, vivienda y recreación, deporte y trabajo, se detallan a continuación en una tabla, algunas de las entidades a donde se puede acudir para información y servicios:

Tabla 4: Entidades que atienden los requerimientos de las PCD

\begin{tabular}{|l|l|}
\hline ÁREA & ENTIDADES DE ATENCIÓN \\
\hline SALUD & $\begin{array}{l}\text { Ministerio de Salud Pública - MSP, Centros Médicos, Municipios en los lugares en donde no tenga el } \\
\text { MSP atención directa, IESS y el de las Fuerzas Armadas }\end{array}$ \\
\hline EDUCACION & $\begin{array}{l}\text { Ministerio de Educación: UDAI Unidades de Apoyo Individual y DECE Departamento de Consejería } \\
\text { Estudiantil }\end{array}$ \\
\hline VIVIENDA Y OTROS & $\begin{array}{l}\text { Ministerio de la Vivienda, Ministerios de Agricultura y Ganadería, Gobierno y Policía, Defensa } \\
\text { Nacional, Ambiente, Energía y Minas }\end{array}$ \\
\hline TRABAJO & Ministerio del Trabajo \\
\hline RECREACIÓN & Municipios, Comunicación, Turismo, INNFA, Organizaciones de PCD, ONG'S \\
\hline
\end{tabular}

Elaborado por: las autoras

\section{Proyectos de apoyo}

Finalmente, para facilitar la lectura, se ha definido como último peldaño de la pirámide establecida para este documento, los proyectos de apoyo encontrados en los documentos públicos. Los beneficios que hasta junio 2016 se ofrecían a las PCD, según una publicación en internet llamada "ecuaconsultas.com", son los que hemos parafraseado en la siguiente tabla: 


\section{Tabla 5: Entidades que atienden los requerimientos de las PCD}

\begin{tabular}{|c|c|c|c|}
\hline ÁREA & TEMA & FUENTE, BASE LEGAL & ENTIDADES RESPONSABLES \\
\hline Impuestos & $\begin{array}{l}\text { Importación y compra de vehículos } \\
\text { Importación de bienes }\end{array}$ & $\begin{array}{l}\text { Ley Orgánica de Discapacidades, publicada en el R.0. №. } 796 \text { de } \\
25 \text { sep. 2012; Arts. 74, } 80 \\
\text { Reglamento de la Ley Orgánica de Discapacidades, publicado en } \\
\text { el R.0. No. } 145 \text { de 17 dic. 2013; Arts. 20, 21,22 } \\
\text { Resoluciones del Servicio Nacional de Aduana del Ecuador-SENAE } \\
\text { - DGN-2014-0111-REy SENAE - DGN-2014-0003-C }\end{array}$ & $\begin{array}{l}\text { Secretaría Nacional de Aduana del Ecuador - SENAE - } \\
\text { www.aduana.gob.ec } \\
\text { Servicio de Rentas Internas -SRI - www.sri.gob.ec }\end{array}$ \\
\hline $\begin{array}{l}\text { Servicio de trans- } \\
\text { porte público }\end{array}$ & Beneficios en valor de transporte & $\begin{array}{l}\text { Acuerdo 006/2008 RO N } 329 \text { / Ley Orgánica de Transporte } \\
\text { Terrestre Tránsito y Seguridad Vial, Art. } 201 \text {. } \\
\text { Resolución U.P.G.T./ Consejo Nacional de Aviación Civil - Codifica- } \\
\text { ción de la Ley, Art. } 55 \text {. - Distrito Metropolitano de Quito - }\end{array}$ & $\begin{array}{l}\text { Agencia Nacional de Tránsito - ANT } \\
\text { www.ant.gob.ec }\end{array}$ \\
\hline $\begin{array}{l}\text { Servicio de trans- } \\
\text { porte público }\end{array}$ & LICENCIA TIP0"F" & Ley Orgánica de Discapacidades - Disposición General Séptima & $\begin{array}{l}\text { Agencia Nacional de Tránsito - ANT / www.ant.gob.ec } \\
\text { Ministerio de Salud Pública - MSP / www.salud.gob.ec }\end{array}$ \\
\hline Impuestos & $\begin{array}{l}\text { Propiedad de vehículos e impuesto } \\
\text { ambiental }\end{array}$ & $\begin{array}{l}\text { Ley Orgánica de Discapacidades. Art. 73. - Reglamento de la Ley } \\
\text { Orgánica de Discapacidades.- Art } 6\end{array}$ & $\begin{array}{l}\text { Servicio de Rentas Internas - SRI } \\
\text { www.sri.gob.ec }\end{array}$ \\
\hline Impuestos & Exención del $50 \%$ impuesto predial & Ley Orgánica de Discapacidades. Art. 75 & Gobiernos autónomos descentralizados municipales \\
\hline Impuestos & A la renta & $\begin{array}{l}\text { Ley Orgánica de Discapacidades. Art. 76. - Reglamento de la Ley } \\
\text { Orgánica de Discapacidades.- Art } 6\end{array}$ & $\begin{array}{l}\text { Servicio de Rentas Internas - SRI } \\
\text { www.sri.gob.ec }\end{array}$ \\
\hline Impuestos & Devolución del IVA & $\begin{array}{l}\text { Reglamento de la Ley Orgánica de Discapacidades. - Disposición } \\
\text { Reformatoria Primera que reforma el Reglamento para la } \\
\text { aplicación de la Ley de Régimen Tributario Interno: Art. } 177\end{array}$ & Servicio de Rentas Internas - SRI \\
\hline Crédito Preferente & Crédito Preferente & $\begin{array}{l}\text { Ley Orgánica de Discapacidades, Art. 55. - Acuerdo Ministerial №. } \\
00142 \text { - MIES de } 11 \text { de noviembre de } 2009\end{array}$ & Banco Nacional de Fomento - BIESS \\
\hline Crédito & $\begin{array}{l}\text { Crédito Desarrollo Humano Asociativo } \\
\text { CDH- IEPS }\end{array}$ & Ley Orgánica de Discapacidades, Art. 55 & $\begin{array}{l}\text { Instituto Ecuatoriano de Economía Popular y Solidaria } \\
\text { www.ieps.gob.ec }\end{array}$ \\
\hline $\begin{array}{l}\text { Crédito } \\
\text { Productivo } \\
\text { Solidario- BDH } \\
\text { - Mies }\end{array}$ & $\begin{array}{l}\text { Crédito de } \$ 840 \text { (menos el } 5 \% \text { que } \\
\text { corresponde a gastos administrativos) }\end{array}$ & $\begin{array}{l}\text { Equivalente a dos años acumulados del BDH que se entrega a las } \\
\text { personas que viven en extrema pobreza. Pueden acceder a los } \\
\text { créditos beneficiarios del bono, pensión para adultos mayores y } \\
\text { pensión para personas con discapacidad. }\end{array}$ & $\begin{array}{l}\text { Ministerio de Inclusión Económica y Social - www. } \\
\text { mies.gob.ec }\end{array}$ \\
\hline Servicios Básicos & Servicios Básicos & Ley Orgánica de Discapacidades, Art. 79 & $\begin{array}{l}\text { Gobiernos autónomos descentralizados / empresas } \\
\text { públicas prestadoras de servicios }\end{array}$ \\
\hline Salud & Afiliación & Ley de Incentivos & Instituto Ecuatoriano de Seguridad Social - IESS \\
\hline Salud & $\begin{array}{l}\text { Jubilación Especial de Vejez por } \\
\text { Discapacidad }\end{array}$ & Ley Orgánica de Discapacidades; Art. 85 & - Instituto Ecuatoriano de Seguridad Social - IESS \\
\hline Salud & Bono "Joaquín Gallegos Lara" & $\begin{array}{l}\text { Decreto Ejecutivo No. } 422 \text { publicado en el R.0. \# } 252 \text { del } 6 \text { de } \\
\text { agosto de } 2010\end{array}$ & $\begin{array}{l}\text { Min. de Inclusión Económica y Social/ SETEDIS http:// } \\
\text { www.setedis.gob.ed/ }\end{array}$ \\
\hline Salud & Bono de Desarrollo Humano & $\begin{array}{l}\text { Decreto Ejecutivo 1395, del } 2 \text { de enero del 2013. Publicado en el } \\
\text { R.0. \#870 del } 14 \text { de enero de } 2013\end{array}$ & $\begin{array}{l}\text { Min. Coordinador de Desarrollo Social / Min de Inclusión } \\
\text { Económica y Social. }\end{array}$ \\
\hline Vivienda & $\begin{array}{l}\text { Incentivo Habitacional (Sistema de incenti- } \\
\text { vos de vivienda rural y urbana marginal) }\end{array}$ & Registro Oficial No. 256 del 12 de agosto de 2010 & Min. de Desarrollo Urbano y Vivienda / SETEDIS \\
\hline Vivienda & $\begin{array}{l}\text { Equipamiento y mejora de Vivienda en } \\
\text { Accesibilidad }\end{array}$ & Ley Orgánica de Discapacidades, Art. 57 & SETEDIS \\
\hline Crédito & Crédito para Vivienda & Ley Orgánica de Discapacidades, Art. 57 & $\begin{array}{l}\text { Banco del Instituto Ecuatoriano de Seguridad } \\
\text { Social - BIESS }\end{array}$ \\
\hline Trabajo & Dotación de Ayudas Técnicas & $\begin{array}{l}\text { Art. 23. Ley Orgánica de Discapacidades Medicamentos, insumos, } \\
\text { ayudas técnicas, producción, disponibilidad y distribución. }\end{array}$ & Ministerio de Salud / SETEDIS \\
\hline Educación & Becas Educativas & Ley Orgánica de Educación Superior, Art. 77. & $\begin{array}{l}\text { Universidades e institutos tecnológicos - IECE } \\
\text { www.lece.gob.ec }\end{array}$ \\
\hline Educación & Capacitación Gratuita SECAP & $\begin{array}{l}\text { - Ley Orgánica de Discapacidades, Art. 54. - Decreto Ejecutivo } 680 \\
\text { - Estructura de Capacitación y Formación Profesional }\end{array}$ & $\begin{array}{l}\text { SECAP Medidas de acción afirmativa. Consejo Nacional } \\
\text { de lgualdad CONADIS }\end{array}$ \\
\hline
\end{tabular}

Elaborado por las autoras, parafraseado de ecuaconsultas.com 
Otro dato relevante en el levantamiento de esta información jurídica, es la creación de los Consejos de Igualdad, instancias creadas en el 2014 por mandato constitucional -transitoria sexta y artículos 156, 157- para reemplazar a los Consejos existentes: Consejo Nacional de las Mujeres (CONAMU), Consejo Nacional de Discapacidades (CONADIS), Consejo Nacional de la Niñez y Adolescencia, CODENPE. Y a su vez, creada a través de cinco Consejos Nacionales para la Igualdad: Género, Intergeneracional, Pueblos y Nacionalidades, Discapacidades y Movilidad Humana, a quienes les informa que se regirán por cinco principios rectores: igualdad, alternabilidad, participación democrática, inclusión, interculturalidad y pluralismo.

A continuación, un gráfico con los proyectos con los que se han comprometido algunas entidades nacionales e internacionales:

Gráfico 4: Compromisos por parte de algunas federaciones para PCD

\section{PROYECTOS DE LAS FEDERACIONES NACIONALES DE Y PARA PERSONAS CON DISCAPACIDAD}

- Proyecto AECIO- COCEMFE

- Proyecto USAID 2010

- SCOUTS

- Capacitación ocupacional e inserción laboral para personas con discapacidad.

- Promoción y exigibilidad de los derechos políticos y laborales de las personas con discapacidad

- Participación ciudadana

- Radio Ecuador Incluyente
Contribuye a la inserción laboral de las personas con discapacidad así como a la sensibilización a empresarios a fin de proveer conocimientos que permitan la formación de espacios laborales amigables para reducir los niveles de deserción laboral.

Guía de Lenguaje Positivo y Comunicación Incluyente

Convenio en favor de la niñez con discapacidad

Mejorar los perfiles ocupacionales de las personas con discapacidad apoyar a la inserción laboral y fomentar la creación de microempresas de personas con discapacidad.

Lograr el involucramiento de las personas con discapacidad en el quehacer político del Ecuador, para que se respete el derecho al voto, a la comunicación e información así como vigilar el cumplimiento de los derechos y la accesibilidad al medio físico.

Fomentar un espacio para construcción de una política de Estado que garantice la observancia y cumplimiento de los derechos humanos.

Radio revista producida por las cinco federaciones nacionales de y para la discapacidad. 
Estos proyectos ofrecen la opción de participar, como ya se dijo, en la formulación, transversalización, observancia, seguimiento y evaluación de las políticas públicas a favor de personas, comunas, comunidades, pueblos, nacionalidades y colectivos, dentro del ámbito de sus competencias relacionadas con las temáticas de género, étnicas, generacionales, interculturales, de discapacidad y movilidad humana, fomentando una cultura de paz que desarrolle capacidades humanas orientadas hacia la garantía del derecho de igualdad y no discriminación.

También les da la potestad de fomentar medidas de acción alternativa y afirmativa que favorezcan la igualdad y la erradicación de actos, usos, prácticas, costumbres y estereotipos considerados discriminatorios.

Cabe señalar que entre los proyectos que sostuvo el gobierno ecuatoriano se desarrolló en 2010, el estudio bio-psicosocial-clínico genético denominado "Misión Solidaria Manuela Espejo", denominándole Sistema de Información Manuela Espejo - SIME, implementado por la Vicepresidencia de la República, determinó que 294.803 personas con discapacidad se encuentran en situación crítica. (Agenda-Nacional-para-Discapacidades, 2016).

También en noviembre del 2016 el periódico "El Universo" publica que el
"Gobierno simplifica trámite para bono Joaquín Gallegos Lara" - JGL, en el que indica que el Ministerio de Inclusión Económica y Social MIES informó de la simplificación de los trámites para que las PCD puedan acceder al bono JGL, comunicando que los requisitos pasarán de 12 a 3: la cédula de ciudadanía, una planilla de servicios básicos y el certificado del MSP. Este subsidio, que corresponde a una transferencia mensual de un salario básico, fue creado en agosto del 2010 por la Vicepresidencia de la República, a través del Decreto Ejecutivo 422, publicado en RO 252 y desde el 2014 es administrado por el MIES. Según datos del gobierno, existen unas 22 mil personas beneficiarias del bono, lo que representa unos 5.2 millones de dólares mensuales entregados a personas con discapacidad severa, a menores de 14 años con VIH-SIDA y a quienes sufran de enfermedades catastróficas raras y huérfanas determinadas por el MSP. (Noticias - Nota 5915113, 2016).

Dentro del Plan Nacional del Buen vivir, se encuentra la Agenda Nacional para Discapacidades 2013 a 2017, que en la resolución del Consejo Nacional de Planificación en el artículo 4 establece lo siguiente:

"Encargar a la Secretaría Nacional de Planificación y Desarrollo la validación de las Agendas Nacionales para la 
Igualdad como insumo de obligatoria observancia para el sector público y para su articulación con los procesos de formulación de política pública de todos los niveles". (Agenda-Nacional-para-Discapacidades, 2016).

Actualmente están registradas cinco Federaciones Nacionales de y para personas con discapacidad: Federación Nacional de Ecuatorianos con Discapacidad Física (FENEDIF); Federación Nacional de Ciegos del Ecuador (FENCE); Federación Nacional de Sordos del Ecuador (FENASEC); y, Federación Ecuatoriana Proatención a la Persona con Deficiencia Mental, Autismo, Parálisis Cerebral y Síndrome de Down (FEPAPDEM), mismas que actualmente, aglutinan a 117 organizaciones de personas con diferente tipo de discapacidad. (CONADIS, 2014).

Tienen cobertura en 21 provincias, las excepciones son: Bolívar, Santa Elena y Galápagos. Su papel fundamental es ofertar servicios a sus organizaciones miembro, personas con discapaci- dad y sus familias, en temas relacionados con capacitación y defensa de derechos humanos, en su gran mayoría; en menor grado en inserción laboral, educación, asesoría, comunicación, servicio de intérpretes de lengua de señas y rehabilitación. Las oficinas de las federaciones funcionan en el CONADIS. (SENADIS, 2017).

\section{Métodos y resultados}

Este estudio se ha enfocado principalmente en recopilar las leyes vigentes en materia de discapacidad, que permitan el análisis de los servicios dispuestos para las PCD y responder a nuestra pregunta: ¿Qué tan complejo resulta para una PCD acceder a las leyes vigentes en torno a sus necesidades y qué tanto se aplican? Por lo tanto, este es un estudio netamente bibliográfico para comprender la situación legal y posteriormente realizar una consulta abierta al público que permita establecer el grado de conocimiento y uso de estos beneficios.

\section{DISCUSIÓN DE RESULTADOS Y CONCLUSIONES}

En este artículo se ha presentado información relevante acerca de los principales organismos y leyes que están destinadas a la atención de PCD. Los derechos inherentes a todo ser huma- no han sido desconocidos por una gran mayoría de la población, especialmente las PCD. Se realizó esta investigación para conocer si existen avances en las acciones que las entidades guberna- 
mentales y jurídicas han ejecutado para el acceso a la participación y ejercicio de deberes y derechos de las PCD. Si bien existe un número importante de ministerios, ONG's, entidades públicas y privadas que componen la acción multidisciplinaria, esto ha permitido de alguna manera articular una serie de actividades necesarias para brindar el servicio y satisfacer las necesidades de las PCD de acuerdo con las diferentes instancias: salud, educación, trabajo y que buscan la manera de mejorar la calidad de vida de los ecuatorianos vulnerables por su discapacidad.

Existen leyes que datan de 1999 y aún se mantienen vigentes, en materia de $P C D$, algunas de las definiciones, obligaciones y procedimientos han variado y existen documentos que lo avalan. Desde el 2003 en el Reglamento a la Ley de discapacidades se indicaban las obligaciones de algunos Ministerios: de Salud, Educación, Bienestar Social (hoy MIES), del Trabajo, Vivienda, Agricultura y Ganadería, Gobierno y Policía, Defensa Nacional, Ambiente, Energía y Minas y otras entidades oficiales como: Comunicación, Turismo, INNFA, Organizaciones de PCD, IESS, ONG's para PCD.

La pregunta que es necesario resolver es si todas las funciones que, el 10 de enero del 2003 se emitieron por Decreto Ejecutivo en el RO 357 que publica el Reglamento General de la Ley de Discapacidades, fueron claramente definidas a los estamentos a quienes se les pasó las atribuciones, en vista que el nuevo Reglamento, expedido el 5 de diciembre del 2013 en DE 171 ya no menciona dichas responsabilidades y deroga el reglamento del 2003. Esto, como es de suponer crea confusión en la comunidad y se obstaculiza la ejecución de los procedimientos requeridos.

Como se puede observar, el Ecuador ha realizado avances importantes en este tema; sin embargo, lo que se ha evidenciado es que no existe una correlación entre los datos que proporcionan los distintos estamentos analizados. En un artículo publicado por la Comisión Económica para América Latina y el Caribe (CEPAL), se encontraron reconocimientos al Ecuador, en cuanto a los avances logrados en la temática relacionada con las PCD.

Pese al camino recorrido, persiste poca coordinación, pues con tanta institución encargada con funciones similares que se trastrocan entre sí, se comprende fácilmente la confusión y poca eficacia que se genera. Al existir un organismo regulador, que en nuestro caso es el CONADIS, debería haber acceso a información veraz. La información y manejo de procesos se confunden, así como las funciones que cada departamento se encarga, pues se encuentran en un sistema burocrático 
complejo y poco amigable. Así se constata al comprobar las cifras y objetivos de cada institución.

Llama la atención, además, la creación de la SETEDIS en el 2013, cuya función era recoger y organizar en un solo ente regulador todo lo mencionado. Sin embargo, tres años después esta entidad fue eliminada. Se concluye entonces, que persiste la desorganización en cada entidad enfocada en la temática. Pues, está visto que la cantidad no garantiza la calidad en la atención. Se evidencia también la falta de información acerca de la existencia de estas organizaciones, factor negativo para el apoyo y desarrollo que las PCD deben lograr.

Entre las conclusiones basadas en las evidencias encontradas, se constata que no hay estadísticas actualizadas y confiables para la elaboración de documentos o futuras investigaciones. A través de la experiencia, se puede ver que aún se los margina de la sociedad y se ha minimizado su participación en el entorno, por ello este escrito lleva de alguna forma a mencionar la necesidad que tienen las PCD que se les dé la relevancia social que se merecen. Ahora, con las nuevas leyes existentes, las PCD pueden cumplir un papel notable en la sociedad puesto que se ha dado ya los inicios para lograr un cambio positivo.

Finalmente, consideramos importante resaltar que la comunidad es la que tiene que educarse, porque de esta forma se puede generar un desarrollo, integración e igualdad de oportunidades en el entorno de una sociedad en donde es prioritario tener presente: "nada sobre nosotros, sin nosotros".

\section{Futuras investigaciones en el tema}

Para futuras investigaciones de este tema, se recomienda realizar una encuesta a las PCD y a sus familias, que permita comprender el alcance y el impacto de las leyes que las protegen. De igual manera se pueden realizar investigaciones cuantitativas que incluyan distintas variables demográficas que permitan obtener datos relevantes en aras de la atención a las necesidades reales individuales y de contexto.

Es necesario trabajar mancomunadamente desde varios sectores políticos y sociales y ofrecer espacios de empoderamiento a las PCD. Quienes estamos en el área de la salud podremos aprovechar los resultados de dicho estudio para hacer cumplir los derechos de las PCD como seres humanos y conocer a dónde se debe acudir.

Es importante recalcar que la discapacidad es un tema de funcionamiento, es decir que más allá del grado o tipo de discapacidad las posibilidades de interactuar y desenvolverse en un medio social es lo que capacita o incapacita a un ser humano. Si los cambios no se rea- 
lizan desde las sociedades en la forma en qué se percibe a la discapacidad, las leyes, acuerdos y disposiciones no bastan para mejorar la calidad de vida de las
PCD. Finalmente, se considera que otro tema de investigación podría centrarse en la mejora de los sistemas de información y comunicación. 


\section{BIBLIOGRAFÎA}

Convención de derechos de las personas con discapacidad. (s.d. de s.d. de 2006). http://www.un.org/esa/socdev/enable/documents/tccconvs. pdf. Recuperado el 15 de 03 de 2017, de http://www.un.org/esa/ socdev/enable/documents/tccconvs.pdf

Agenda-Nacional-para-Discapacidades. (s.d. de s.d. de 2016). Agenda-Nacional-para-Discapacidades. . Recuperado el 08 de 03 de 2017, de Sitio web de: Planificación: http://www.planificacion.gob. ec/wp-content/uploads/downloads/2014/09/.pd

Asamblea Nacional del Ecuador. (2008). Constitución de la República del Ecuador. Quito: Ediciones Legales. Asamblea Nacional del Ecuador. (2012). Ley Orgánica de Discapacidades LOD. Quito: Ediciones Legales, RO 796.

Asamblea Nacional, Registro Oficial N. 796. (s.d. de 10 de 2012). Ley Orgánica de Discapacidades - LOD. Recuperado el 15 de 03 de 2017, de Sitio de CONADIS: http://www. conadis.gob.ec/images/documentos/nuevaleyorganicadediscapacidades.pdf

CEPAL - Bernal M.E. y Valencia C. (s.d. de marzo de 2016). Institucionalidad y marco legislativo de la discapacidad en el Ecuador. Recuperado el 25 de 03 de 2017, de Sitio de CEPAL - Comisión Económica para América Latina y el Caribe: http:// repositorio.cepal.org

CONADIS. (2014). Libro Normas Jurídicas en Discapacidad Ecuador. Quito: Imprenta Don Bosco.

CONADIS- Normas jurídicas en Discapacidad Ecuador. (30 de mayo de 2012). http://plataformaconadis.gob.ec/wp-content/ uploads/2014/08/Libro-Normas-Juridicas-en-Discapacidad-Ecuador.pdf. Obtenido de http://plataformaconadis.gob.ec/ wp-content/uploads/2014/08/ Libro-Normas-Juridicas-en-Discapacidad-Ecuador.pdf

Noticias - Nota 5915113. (21 de 11 de 2016). Gobierno-simpifica-tramite-bono-Joaquin-Gallegos-Lara. El Universo, pág. http:// www.eluniverso.com/noticias/ nota/5915113/.

OMS - OPS. (2001). Clasificación Internacional del Funcionamiento de la Discapacidad y de la Salud: CIF. Madrid: Grafo S.A.

Organización de las Naciones Unidas. (s.d. de s.d. de s.d.). ONU - Declaración de los Derechos Humanos. 
Recuperado el 08 de 03 de 2017, de Sitio web de ORG: http:// www.un.org/es/rights/overview: http://www.un.org/es/sections/ issues-depth/human-rights/index.html

Organización Mundial de la Salud. (s.d. de s.d. de 2016). Tema de salud: Discapacidades. Recuperado el 10 de 03 de 2017, de Sitio web de OMS: http://www.who.int/topics/ disabilities/es/

SENADIS. (s.d. de s.d. de 2017). http:// www.senadis.gob.pa/?page_ $i d=10$. Recuperado el 12 de 03 de 2017, de Sitio de CONADIS: http://www.senadis.gob.pa/?page_id $=10$

Toboso, M. y. (2008). La discapacidad dentro del enfoque de capacidades y funcionamientos de Amartya Sen. Madrid: s.d.

Verdugo A. M. (1998). Personas con discapacidad. Madrid: Siglo Veintiuno de España Editores.

Verdugo M.A. Schalock R. . (2013). Discapacidad e Inclusión. Madrid: Ediciones Amarú. 